\title{
Autoimmune Disease-Associated Variants of Extracellular Endoplasmic Reticulum Aminopeptidase 1 Induce Altered Innate Immune Responses by Human Immune Cells
}

\author{
Yasser A. Aldhamen ${ }^{a} \quad$ Yuliya Pepelyayeva $^{\text {a }}$ David P.W. Rastall ${ }^{a}$ \\ Sergey S. Seregin ${ }^{a}$ Efthalia Zervoudic Despoina Koumantou ${ }^{c}$ \\ Charles F. Aylsworth $^{\mathrm{a}}$ Dionisia Quiroga ${ }^{\mathrm{a}}$ Sarah Godbehere ${ }^{\mathrm{a}}$ \\ Dimitris Georgiadis $^{d}$ Efstratios Stratikos ${ }^{c}$ Andrea Amalfitano ${ }^{a, b}$ \\ Departments of a Microbiology and Molecular Genetics and ${ }^{b}$ Pediatrics, College of Osteopathic Medicine, \\ Michigan State University, East Lansing, Mich., USA; ${ }^{C}$ Protein Chemistry Laboratory, National Center for Scientific \\ Research Demokritos, and d Department of Chemistry, Laboratory of Organic Chemistry, University of Athens, \\ Athens, Greece
}

\section{Key Words}

Autoimmunity · Endoplasmic reticulum aminopeptidase 1 . Caspase-1 1 Interleukin-1 $\beta$. Adenovirus

\begin{abstract}
Endoplasmic reticulum aminopeptidase 1 (ERAP1) gene polymorphisms have been linked to several autoimmune diseases; however, the molecular mechanisms underlying these associations are not well understood. Recently, we demonstrated that ERAP1 regulates key aspects of the innate immune response. Previous studies show ERAP1 to be endoplasmic reticulum-localized and secreted during inflammation. Herein, we investigate the possible roles that ERAP1 polymorphic variants may have in modulating the innate immune responses of human peripheral blood mononuclear cells (hPBMCs) using two experimental methods: extracellular exposure of hPBMCs to ERAP1 variants and adenovirus (Ad)-based ERAP1 expression. We found that exposure of hPBMCs to ERAP1 variant proteins as well as ERAP1 overexpression by Ad5 vectors increased inflamma-
\end{abstract}

tory cytokine and chemokine production, and enhanced immune cell activation. Investigating the molecular mechanisms behind these responses revealed that ERAP1 is able to activate innate immunity via multiple pathways, including the NLRP3 (NOD-like receptor, pyrin domain-containing 3) inflammasome. Importantly, these responses varied if autoimmune disease-associated variants of ERAP1 were examined in the assay systems. Unexpectedly, blocking ERAP1 cellular internalization augmented IL-1 $\beta$ production. To our knowledge, this is the first report identifying ERAP1 as being involved in modulating innate responses of human immune cells, a finding that may explain why ERAP1 has been genetically associated with several autoimmune diseases.

(c) 2015 S. Karger AG, Basel

\section{Introduction}

Autoimmune and autoinflammatory diseases are a heterogeneous group of disorders that affect various organ systems in the body [1]. These disorders are charac-

\section{KARGER 125}

2015 S. Karger AG, Base

$1662-811 X / 15 / 0073-0275 \$ 39.50 / 0$

E-Mail karger@karger.com

www.karger.com/jin
Dr. Andrea Amalfitano

Department of Microbiology and Molecular Genetics

Michigan State University, 4194 Biomedical and Physical Sciences Building

East Lansing, MI 48823 (USA)

E-Mail amalfit1@msu.edu 
terized by abnormally enhanced proinflammatory immune responses primarily mediated by the cells and molecules of the innate immune system [2]. In addition, both autoimmune and autoinflammatory diseases have been shown to have phenotypes which are associated with chronic activation of innate and adaptive immune system components, and often resulting in tissue inflammation and damage, especially in genetically predisposed individuals [3].

The innate immune system is the first line of host defense against invading pathogens. It relies heavily on a variety of transmembrane, intracellular and secreted pattern-recognition receptors, each of which are vital for the recognition of specific molecular structures found on, or within, potentially infectious agents such as viruses or microbes [4]. Activation of pattern-recognition receptors like Toll-like receptors, RIG-I-like receptors and NODlike receptors drive the coordinated activation of various signaling pathways that regulate the transcription of proinflammatory cytokine and chemokine genes as well as other innate immune defense responses. These responses are essential for shaping the subsequent, antigen-specific adaptive immune responses [5].

Many important immune genes have been shown to play a role in autoimmunity and it is widely appreciated that allelic variations within these genes can differentially drive self-reactive immune responses and lead to the development of autoimmune diseases [6]. As confirmed by multiple genome-wide association studies, endoplasmic reticulum aminopeptidase 1 (ERAP1) has been identified as being one of these genes [7].

ERAP1 is an IFN $\gamma$ - and TNFa-inducible, ubiquitously-expressed, zinc-dependent, endoplasmic reticulumlocalized aminopeptidase that belongs to the oxytocinase subfamily of M1 aminopeptidases [8]. Several variants of the ERAP1 gene have been linked to human diseases [9], with the strongest genetic associations being found in patients with the chronic inflammatory disease ankylosing spondylitis (AS). ERAP1 polymorphisms are responsible for approximately $26 \%$ of the population-attributed risk for AS [10]. Moreover, allelic variants of ERAP1 have been linked to other pathological conditions such as type I diabetes, multiple sclerosis, cervical cancer, Crohn's disease, Behçet's disease and hypertension [7], suggesting that ERAP1 polymorphisms can have diverse effects. In addition, allelic variants of the human ERAP1 gene have been shown to occur as functionally distinct haplotypes [11]. Of the known ERAP1 single-nucleotide polymorphisms (SNPs), 2 have been repeatedly confirmed to confer strong susceptibility to autoimmune disease: rs30187
(K528R or ERAP1-RQ) and rs27044 (Q730E or ERAP1$\mathrm{KE}$ [7]. In particular, ERAP1-RQ has been the primary variant reported to be frequently associated with AS, type I diabetes and multiple sclerosis [12]. We have previously produced recombinant forms of these 2 variants and have shown significant differences in their enzymatic activities compared to other ERAP1 polymorphisms [13]. Moreover, we and other study groups have shown that the AS-associated ERAP1 SNPs significantly alter ERAP1 functions, including antigen-presentation [14].

Although these and other studies suggest that the association of ERAP1 to autoimmune diseases may be primarily attributed to its role in MHC class I antigen presentation [15], we have found that ERAP1 also possesses non-MHC immune functions, such as regulating innate immune responses during the initial stages of pathogen recognition in vivo [16]. Specifically, we demonstrated that, relative to wild-type mice, mice lacking ERAP1 exhibit exaggerated innate immune responses during early pathogen recognition, as characterized by the increased activation of natural killer (NK) and NK T cells and the enhanced production of proinflammatory cytokines and chemokines such as IL-6, IL-12, TNFa and MCP-1 [16]. We also found that ERAP1 plays a critical role in regulating early NK cell development and function, as shown by the higher frequencies of terminally matured and licensed NK cells present in ERAP1-knockout mice. These results positively correlated with enhanced NK activation and IFN $\gamma$ production by cells derived from ERAP1-knockout mice, suggesting ERAP1 has a critical role in the modulation of innate immune responses during the initial stages of pathogen recognition. In this study, with the use of hPBMCs, we investigate the possible roles that the autoimmune disease-associated ERAP1 variants may have in differentially modulating innate immune responses.

\section{Materials and Methods}

Adenovirus Vector Construction, Production and Characterization

The Ad5-Null virus was purified as previously described [17]. For Ad5-ERAP1_high and Ad5-ERAP1_low vectors, pShuttle plasmids contained a CMV expression cassette and a SV40-derived polyadenylation signal flanking either the high-risk ERAP1 (349M, 528K, 575D, 725R and 730Q amino acid SNPs) or the lowrisk ERAP1 (349V, 528R, 575N, 725Q and 730E amino acid SNPs) alleles, respectively, and were constructed by bacterial subcloning as previously described [18]. The resulting pShuttle-ERAP1_high and pShuttle-ERAP1_low plasmids were linearized with PmeI restriction enzyme and homologously recombined with the pAdEasyI Ad5 vector genome to yield pAd-ERAP1_high and pAd-ERAP1_ 
low, as previously described [18]. HEK293 cells were transfected with the PacI-linearized plasmids and viable viruses were obtained and amplified after several rounds of expanding infection. Ad viruses were purified using a $\mathrm{CsCl}_{2}$ gradient, as previously described [17]. Direct sequencing and restriction enzyme mapping were carried out to confirm the integrity of the ERAP1 sequence. All viruses were found to be free of replication-competent adenovirus, by replication-competent adenovirus PCR (E1 region amplification) and direct-sequencing methods, as previously described [17]. Due to the lack of the Ad5-specific receptor CAR (coxsackie adenoviral receptor) on hPBMCs, increased doses of the respective Ad5 vectors were utilized in this study, as previously described [19, $20]$ in our studies investigating the impact of ERAP1 overexpression on the innate immune responses of hPBMCs. ERAP1-expressing or control Ad5 vectors were used at a multiplicity of infection of 10,000 viral particles per cell, a viral dose that allows sufficient gene transfer to induce a dramatic increases in transgene expression without deleterious effects to the Ad5-transduced cells $[21,22]$.

\section{Protein Expression and Purification}

At least 3 different preparations of each ERAP1 protein were utilized in our studies to confirm that the results obtained could be repeatable and not dependent on uncontrollable issues during protein purification. The generation of the ERAP1 variant-expressing pDEST8 vectors has been described before [23]. We utilized 4 ERAP1 variants: ERAP1-528K/730Q (ERAP1-KQ), ERAP1K528R (ERAP1-RQ), ERAP1-Q730E (ERAP1-KE) and ERAP1K528R/Q730E (ERAP1-RE). Recombinant baculovirus containing each ERAP1 variant was produced in SF9 cells using a Bac-toBac baculovirus expression system, as per the manufacturer's instructions (Invitrogen). Recombinant proteins were expressed in Hi5 cells after recombinant baculovirus infection and purified as previously described [24]. Proteins were aliquoted and stored in a buffer containing $20 \mathrm{mM}$ HEPES (pH 7), $100 \mathrm{mM} \mathrm{NaCl}$ and $10 \%$ glycerol at $-80^{\circ} \mathrm{C}$. Enzymatic activity was verified by small fluorigenic substrate hydrolysis, as previously described [24]. The ERAP1 inhibitors DG002A and DG013A were synthesized and purified as described [25]. All hPBMC experiments utilizing ERAP1 protein were carried out using $500 \mathrm{ng}$ of ERAP1, unless otherwise specified.

\section{Isolation of $h P B M C s$}

Human PBMCs were isolated from fresh buffy coat material (Stanford Blood Center, Palo Alto, Calif., USA) with standard techniques using either Histopaque 1077 (Sigma St. Louis, Mo., USA) or Lympholyte H (Cedarlane Diagnostics, Ontario, Canada), according to the manufacturer's protocol. All blood samples, obtained from the Stanford Blood Center, were deidentified and a waiver was obtained from the Michigan State University IRB office. At least 8 different buffy coats were utilized in our studies, allowing us to ascertain whether variations from human to human might significantly impact the results of our studies. Briefly, buffy coat material was diluted 1:1 in PBS, layered onto Histopaque 1077 (or Lympholyte H), and centrifuged at room temperature for $30 \mathrm{~min}$ at $400 \mathrm{~g}$. Cells sedimenting at the interface were removed and washed extensively with PBS to remove remaining platelets. Isolated hPBMCs were quantified, diluted, and cultured in 5\% FBS containing RPMI 1640 supplemented with human IL-2 (5 ng/ml) and human IL-18 (10 ng/ml) because these cytokines allow for minor activation of human immune cells and enhanced proinflammatory stimuli responses [22].

\section{Cell Staining and Flow Cytometry}

Human PBMCs $\left(2 \times 10^{6}\right)$ were harvested and initially stained with purified mouse anti-human CD16/CD32 Fc $\gamma$ block, followed by APC-Cy7-CD3, APC-CD56, APC-Cy7-CD1a, Alexa Fluor700CD14 and Pacific blue CD80 (all at $4 \mu \mathrm{g} / \mathrm{ml}$; BD Biosciences, San Diego, Calif., USA). Cells were incubated on ice with the appropriate antibodies for $45 \mathrm{~min}$ and washed with FACS buffer. A BD LSR II instrument was used for data collection and data were analyzed using FlowJo software.

\section{Reagents}

FAM-FLICA in vitro caspase-1 detection kit was purchased from Immunochemistry Technologies (Bloomington, Minn., USA). CA-074 methyl ester (CA-074Me) was from Calbiochem (EMD Millipore, Billerica, Mass., USA). Alum was purchased from Thermoscientific (Waltham, Mass., USA). Cytochalasin D, adenosine triphosphate (ATP) and lipopolysaccharide (LPS) were from Sigma-Aldrich (St. Louis, Mo., USA). The caspase-1 inhibitor z-YVAD-FMK was purchased from Promega (Madison, Wisc., USA). EDTA was from EMD Millipore.

\section{ELISA}

Cell culture supernatants were assayed for the concentration of IL-1 $\beta$, IL-6, and TNF $\alpha$ with ELISA kits from BD Biosciences according to the manufacturer's instructions. Briefly, cells were treated with various ERAP1 variants for 24 or $48 \mathrm{~h}$ and supernatants were collected and assayed for cytokine secretion.

\section{Cytokine and Chemokine Analysis}

A human 27-plex multiplex-based assay was used to determine cytokine/chemokine concentrations of selected supernatant media collected from hPBMC cultures per the manufacturer's instructions (Bio-Rad, Hercules, Calif., USA) via Luminex 100 technology (Luminex, Austin, Tex., USA).

\section{Phagocytosis Assay}

Murine macrophage RAW264.7 cells were cultured in RPMI 1640 containing $10 \%$ heat-inactivated fetal bovine serum. For assays, cells were transferred and cultured in 96-well black culture plates $\left(2 \times 10^{4}\right.$ cells/well $)$ for $24 \mathrm{~h}$ at $37^{\circ} \mathrm{C}$ and $5 \% \mathrm{CO}_{2}$. The next day, they were washed twice with cold phosphate-buffered saline (PBS) and activated with IFN $\gamma(100 \mathrm{IU} / \mathrm{ml})$ and LPS $(1 \mu \mathrm{g} / \mathrm{ml})$ in the presence or absence of various inhibitor concentrations. Phagocytosis was assessed by measuring the amount of FITC-labeled rabbit IgG latex bead uptake into cells using a phagocytosis assay kit, (Cayman Chemical, Ann Arbor, Mich., USA) according to the instruction manual. In brief, cells with or without various stimulants were treated with FITC-beads and cultured in RPMI 1640 with $1 \% \mathrm{FBS}$ for $24 \mathrm{~h}$ at $37^{\circ} \mathrm{C}$ and $5 \% \mathrm{CO}_{2}$. Twenty-four hours after stimulation, cells were washed twice with cold PBS. Cellularbead uptake was calculated by measuring fluorescence intensity in a TECAN infinite ${ }^{\circledR}$ M200 microplate-fluorescence reader using an excitation of $485 \mathrm{~nm}$ and emission of $535 \mathrm{~nm}$.

Cell supernatant from treated cells was used for measuring aminopeptidase activity. Enzymatic activity of ERAP1 was determined with L-leucine-7-amido-4-methyl coumarin (Leu-AMC; 
Sigma). The reaction mixture containing $100 \mu \mathrm{M}$ Leu-AMC and $50 \mu$ of culture medium in $50 \mathrm{~mm}$ HEPES pH 7.0 and $100 \mathrm{mM} \mathrm{NaCl}$ was added to a 96-well black plate. The amount of AMC released was measured by a TECAN infinite M200 microplate-fluorescence reader at an excitation wavelength of $380 \mathrm{~nm}$ and emission wavelength of $460 \mathrm{~nm}$.

\section{Statistical Analysis}

Differences in innate immune responses were determined using one-way ANOVA with the Student-Newman-Keuls post hoc test or by using the two-tailed homoscedastic Student $t$ test, with a $p$ value $<0.05$ denoting statistical significance. All graphs are presented as mean \pm standard error of the mean, unless otherwise specified. GraphPad Prism software was utilized for statistical analysis.

\section{Results}

\section{Extracellular ERAP1 Protein Induces}

Proinflammatory Cytokine and Chemokine Secretion in an Allele-Dependent Fashion

Previous reports have shown that ERAP1 protein is secreted from macrophages in response to LPS and IFN $\gamma$ stimulation, and treatment with ERAP1-containing supernatants enhances macrophage phagocytosis [26]. However, the exact molecular mechanism underlying these observations has yet to be defined. To expand upon these previous studies and correlate them with human disease pathogenesis, we wished to test if ERAP1 autoimmune disease-associated SNP-containing variants may differentially engage the innate immune regulatory functions of human immune cells. Disease-associated ERAP1-RQ and ERAP1-KE variants were utilized alongside the protective ancestral reference variant ERAP1-KQ (sequence NP_057526) and double-polymorphism-carrying variant ERAP1-RE (K528R, Q730E) [13]. Human PBMCs were incubated with recombinant ERAP1 proteins and, following $48 \mathrm{~h}$ incubation, cell media were collected to measure inflammatory cytokines and chemokine secretion. We also investigated if these SNPs function synergistically or antagonistically by including ERAP1 variants that carry individual SNPs in our analysis.

Our results demonstrated that hPBMCs treated with ERAP1 produce markedly increased levels of several inflammatory cytokines and chemokines in a dose-dependent manner compared to mock-treated controls (fig. 1). Specifically, we observed a more potent production of IL$1 \beta$ ( 5- and 20-fold), IL-6 ( 1.5- and 2-fold) and TNFa ( $\sim 1-$ and 4 -fold) in cells treated with 0.5 and $1 \mu \mathrm{g}$ of ERAP1-RQ compared to cells treated with other ERAP1 variants (fig. 1a-c). Treatment with the ERAP1-KE variant also induced higher cytokine levels than the ERAP1KQ and ERAP1-RE variants. Interestingly, exposure of hPBMCs to the ERAP1-KQ protein failed to induce detectable levels of IL- $1 \beta$, IL- 6 and TNF $\alpha$, a result consistent with previous genome-wide association studies that showed the ERAP1-KQ variant to be protective against various autoimmune diseases [7].

Importantly, exposure of hPBMCs to ERAP1-KE induced the potent production of multiple cytokines and chemokines compared to both mock-treated and control protein-treated cells (online suppl. fig. 1A-C and table 1; for all online suppl. material, see www.karger.com/ doi/10.1159/000368899). To exclude the possible confounding effects of contaminating endotoxins in the ERAP1 protein preparations, polymyxin B preincubation was performed before ERAP1 stimulations. Polymyxin B was found to significantly reduce LPS-mediated TNFa and IL-6 release, but had no significant effect on ERAP1dependent cytokine release treated cells (online suppl. fig. 2).

To further demonstrate that ERAP1 was responsible for these responses, we utilized an adenovirus (Ad)5based expression system to upregulate the production of different ERAP1 variants intracellularly. We utilized two recombinant Ad5 vectors, the first expressing a high-disease-risk ERAP1 allele (Ad5-ERAP1_high) and the other expressing a disease-protective ERAP1 allele (Ad5ERAP1_low). Human PBMCs were infected with a MOI of 10,000 viral particles of either the experimental or control Ad5 vectors, as previously described [21, 22]. Consistent with our previously published data [21], Ad5 vector infection of hPBMCs induced the production of several inflammatory cytokines and chemokines when compared to mock-treated controls (fig. 2a-c and online suppl. table 2). Moreover, ERAP1 overexpression further enhances these responses when compared to cells infected with the Ad5-Null controls (fig. $2 \mathrm{a}-\mathrm{c}$ and online suppl. table 2). In particular, significantly $(\mathrm{p}<0.001)$ enhanced production levels of IL-1 $\beta$, IL- 6 and TNF $\alpha$ were observed in cells infected with Ad5-ERAP1_high and Ad5-ERAP1_ low when compared to cells infected with the Ad5-Null controls (fig. 2a-c). Interestingly, we observed dramatic increases (approx. 4-fold) of IL- $1 \beta$ production from Ad5ERAP1_high-infected hPBMCs compared to cells infected with the Ad5-ERAP1_low expressing Ad vector (fig. 2a). These data demonstrate that disease-linked ERAP1 variants can induce differential cytokine and chemokine responses and possess critical innate immunostimulatory activity. 


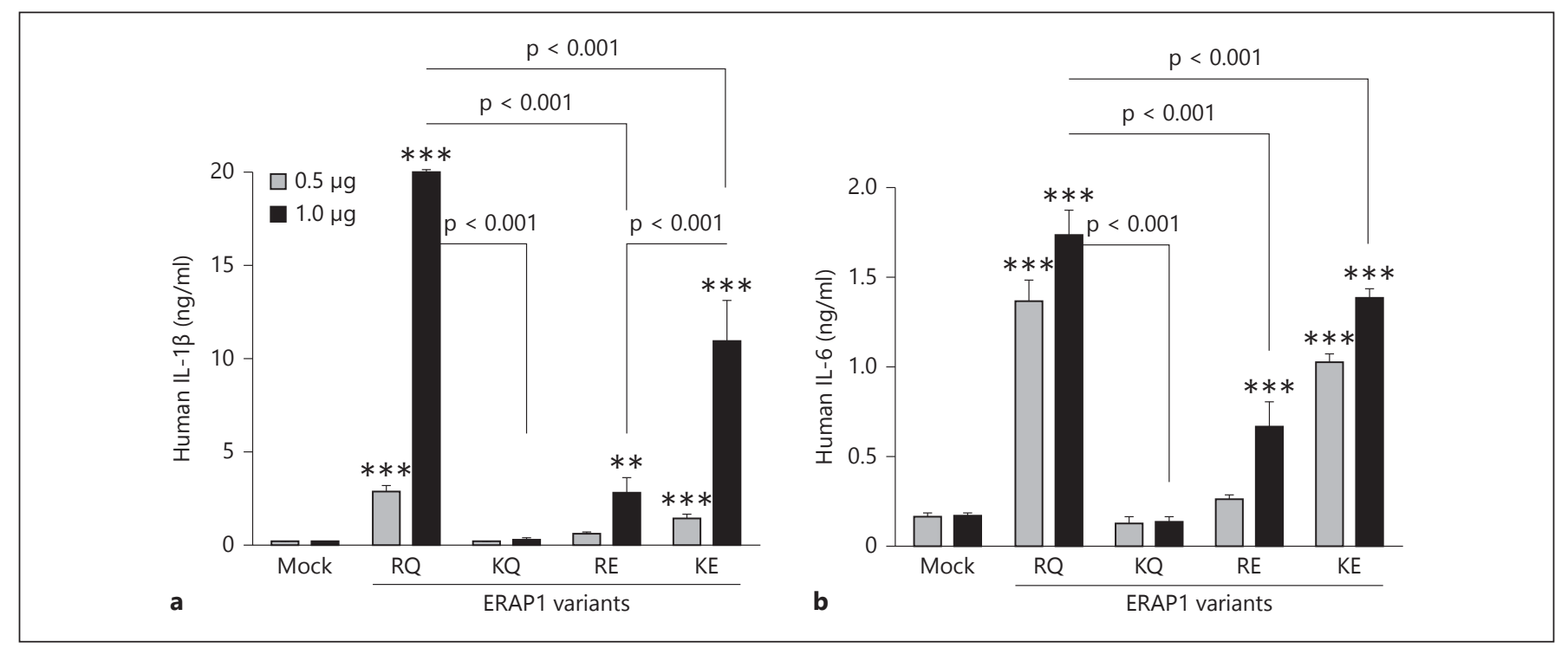

Fig. 1. Differential production of inflammatory cytokines and chemokines following treatment with several ERAP1 variants: hPBMCs $\left(2 \times 10^{6}\right.$ cells/well $)$ were either mock-treated or treated with an ERAP1 protein variant (ERAP1-RQ, ERAP1-KQ, ERAP1-RE and ERAP1-KE). Culture media were collected at $48 \mathrm{~h}$ post treatment and analyzed for cytokines and chemokines levels. ELISA of the production of IL-1 $\beta$ (a), IL-6 (b) and TNFa (c) by hPBMCs are shown. Samples were plated in quadruplicate. Data are representative of 2 independent experiments for the comparison of all ERAP1 variants and at least 6 independent experiments from 6 different buffy coat donors for ERAP1-RQ and ERAP1-KE variants. ${ }^{* *} \mathrm{p}<$ $0.01,{ }^{* * *} \mathrm{p}<0.001$ denote statistical significance from mock-treated cells.
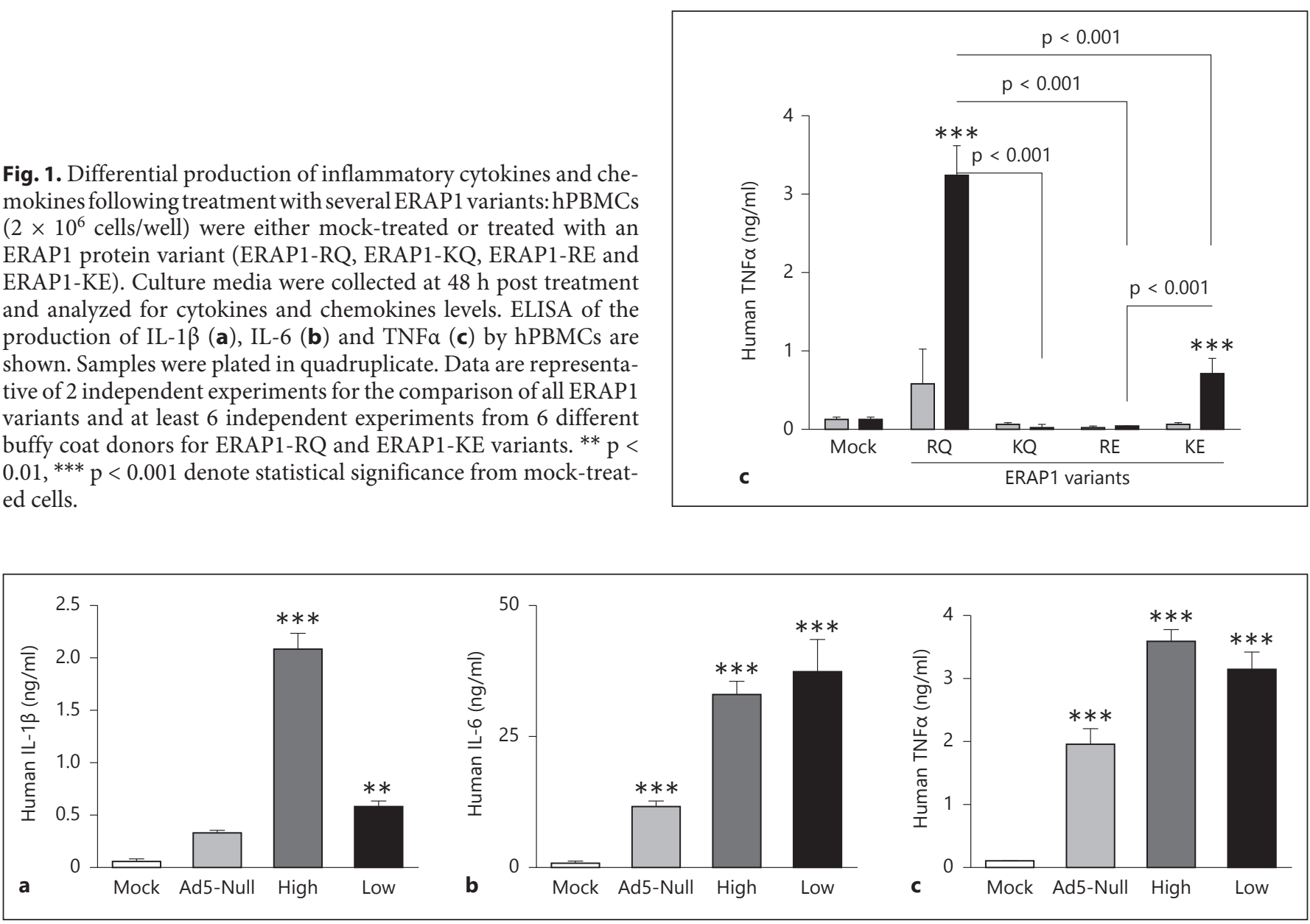

Fig. 2. ERAP1 overexpression induces inflammatory cytokine and chemokine production: hPBMCs $\left(2 \times 10^{6}\right.$ cells/well $)$ were either left uninfected or infected with Ad5-ERAP1_high (High), Ad5ERAP1_low (Low) or Ad5-Null control (a multiplicity of infection of 10,000 viral particles/cell). After 48-hour incubation, media were collected to perform a bioplex bead array to measure cytokine and chemokine responses. Secretion of IL-1 $\beta$ (a), IL-6 (b) and TNFa (c) by hPBMCs is shown. Data are representative of 2 independent experiments from 2 different buffy coat donors. ${ }^{* *} \mathrm{p}<0.01$, ${ }^{* * *} \mathrm{p}<$ 0.001 denote statistical significance from mock-treated cells. 
Extracellular ERAP1 Protein Activates Human Innate and Adaptive Immune Cells

To investigate if ERAP1 alterations in cytokine secretion affect innate immune cell functions, the phenotype of human immune cells exposed to extracellular ERAP1 protein variants was investigated by flow cytometry analysis. We observed significantly increased levels of human NK cells activation following ERAP1 treatment, as evidenced by increased CD107a expression on CD56+CD3cells, as compared to mock-treated cells (fig. $3 a$ ). In addition, a more potent expression of CD107a was observed on NK cells exposed to ERAP1-RQ compared to cells treated with other ERAP1 variants (fig. 3a, b). Significant activation of human NK cells was also observed after ERAP1-KE and ERAP1-RE exposure compared to mock-treated control cells (fig. 3a, b). In contrast, ERAP1-KQ did not induce a significant increase of CD107a expression on NK cells compared to mocktreated cells (fig. $3 a, b)$.

Similar results were also observed on $\mathrm{CD} 3+\mathrm{T}$ cells derived from ERAP1-treated hPBMCs, as there was a significantly increased expression of CD69 and CD107 $\alpha$ observed on T cells treated with ERAP1-RQ, ERAP1-KE or ERAP1-RE, but not ERAP1-KQ, when compared to mock-treated cells (fig. 3c, d). To exclude the possibility that these results were due to LPS contamination of the respective ERAP1 proteins, we also evaluated the activation of T cells and NK cells following ERAP1 treatment in the presence of the LPS neutralizing agent, polymyxin B. Polymyxin B pretreatment significantly reduced the LPS-mediated T cell (online suppl. fig. 2C) and NK cell (online suppl. fig. 2D) activation whereas polymyxin $\mathrm{B}$ treatment of ERAP1 treated cells had no significant effect (online suppl. fig. 2C, D).

To further verify these results, we repeated the experiments in the presence of DG013A and DG002A, previously described ERAP1 inhibitors [25]. Enhanced NK cell activation induced by ERAP1-RQ treatment was com-

Fig. 3. Differential activation of human innate and adaptive immune cells by the ERAP1 variants and ERAP1 enzymatic activity are required for these responses: hPBMCs $\left(2 \times 10^{6}\right.$ cells/well $)$ were either mock-treated or treated with $1 \mu \mathrm{g}$ of an ERAP1 protein variant. Cells were harvested at $48 \mathrm{~h}$ after treatment for flow cytometry analysis. a A representative example of the gating strategy used to define the frequency of CD107a-expressing, CD56+CD3- human NK cells. Gates were set based on mock-treatment levels and placed consistently across samples. b Expression of CD107a on human NK cells. Expression of CD69 (c) or CD107a (d) on human CD3 + T cells was also measured. e Expression of CD107 $\alpha$ on CD56+CD3- NK cells treated with the ERAP1-RQ in the presence pletely abolished following ERAP1 inhibitor treatment (fig. 3e), and similar results were observed when CD80 expression on human dendritic cells (DCs) was evaluated (fig. 3f). Finally, to determine if these effects are dependent on known ERAP1 aminopeptidase enzymatic activities, we pretreated recombinant ERAP1 with the zincchelating agent EDTA to abolish its enzymatic activity [27]. Human PBMCs were treated with intact or EDTA (5 mM)-treated ERAP1-RQ protein and $48 \mathrm{~h}$ after treatment, media were collected for the measurement of IL$1 \beta$, IL- 6 and TNFa secretion by ELISA. Consistent with the above results, treatment with intact ERAP1-RQ induced potent IL- $1 \beta$ production, while EDTA treatment of ERAP1-RQ protein completely abrogated IL- $1 \beta$ secretion (fig. 3g), suggesting that the aminopeptidase enzymatic activity of ERAP1 plays a direct role in mediating these responses. We observed similar results for IL-6 (fig. 3h) and TNFa (fig. 3i).

\section{Preventing Extracellular ERAP1 Trimming Activity}

Alters ERAP1-Mediated Innate Immune Responses

Previously, it has been shown that IFN $\gamma$ and LPS treatment of RAW264.7 macrophages induces secretion of ERAP1 and that the ERAP1-containing supernatant from these cells can enhance macrophage phagocytosis [26]. However, it is not clear how ERAP1 mediates these responses. To investigate if extracellular ERAP1 exerts proinflammatory effects via characterized peptide-trimming activities, we treated RAW264.7 cells with IFN $\gamma$ $(100 \mathrm{IU} / \mathrm{ml})$ and LPS $(1 \mu \mathrm{g} / \mathrm{ml})$ and evaluated macrophage phagocytosis in the presence of escalating doses of our recently identified ERAP1 inhibitor, DG013A [25]. Interestingly, we not only verified the ability of extracellular ERAP1 to induce macrophage phagocytosis, but also found that pretreatment of macrophages with DG013A significantly ablates the macrophage phagocytosis activity of ERAP1 in a dose-dependent manner (fig. 4a). To confirm that the secretory autocrine/para-

or absence of $5 \mu \mathrm{M}$ of DG013A and DG002A was measured. $\mathbf{f}$ Expression of CD80 on CD1 $\alpha+C D 14-$ hPBMC-derived DCs treated with ERAP1-RQ in the presence or absence of $5 \mu \mathrm{M}$ DG002A was quantified. Samples were plated in quadruplicate. IL-1 $\beta$ (g), IL-6 (h) and TNFa (i) production by hPBMCs treated with $500 \mathrm{ng}$ of the intact or $5 \mathrm{mM}$ EDTA-inactivated ERAP1-RQ protein was performed using media collected at time of cell harvest. Data are representative of at least $3(\mathbf{a}-\mathbf{c}, \mathbf{e}, \mathbf{f})$ or $2(\mathbf{d})$ independent experiments from different blood donors. Cells were plated in quadruplicate. ${ }^{*} \mathrm{p}<0.05,{ }^{* *} \mathrm{p}<0.01,{ }^{* * *} \mathrm{p}<0.001$ denote statistical significance from mock-treated cells.

(For figure see next page.) 


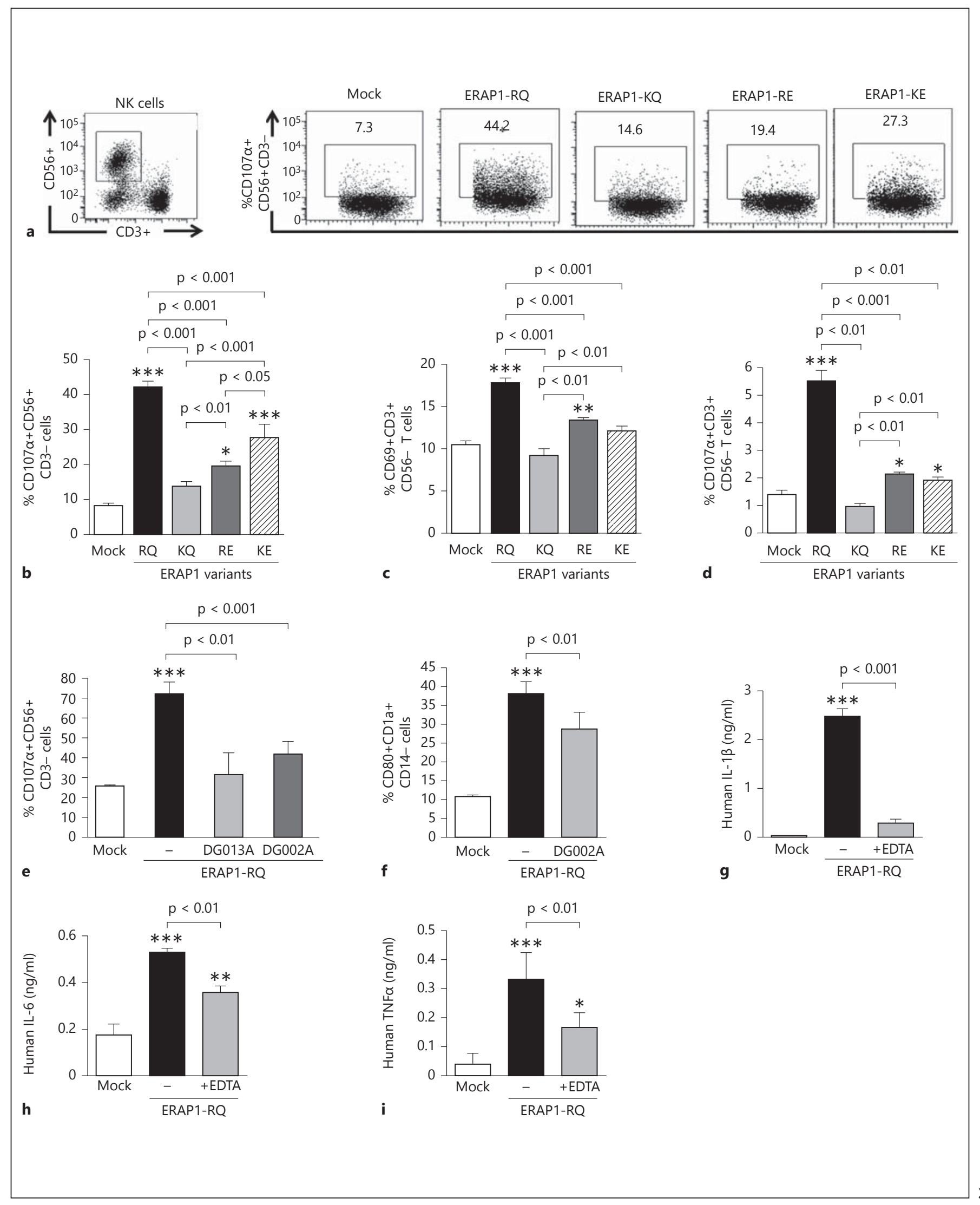



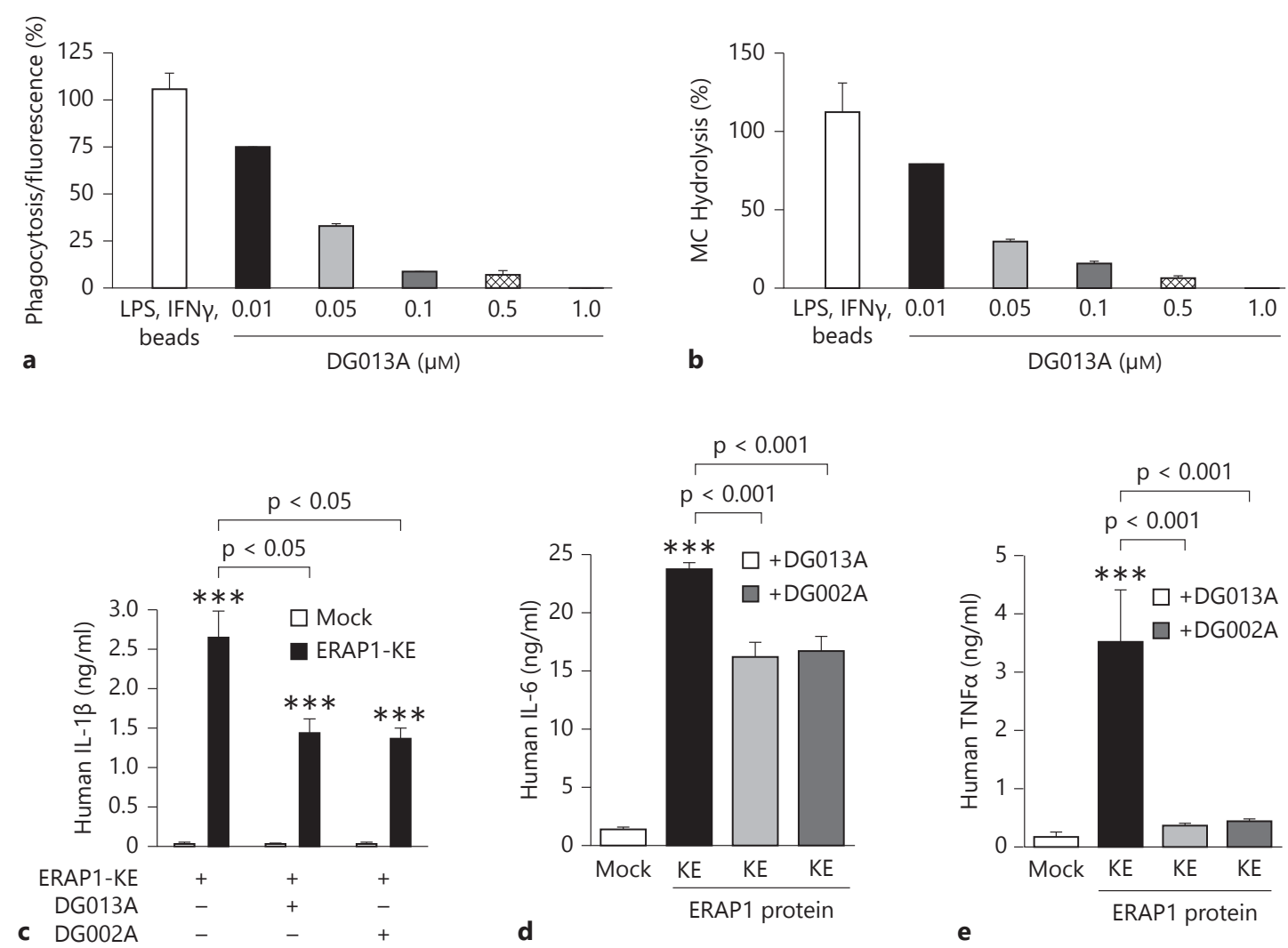

Fig. 4. Preventing extracellular ERAP1 trimming activity alters ERAP1-mediated innate immune responses: LPS $(1 \mu \mathrm{g} / \mathrm{ml}) / \mathrm{IFN} \gamma$ (100 IU/ml)-treated RAW264.7 cells were incubated for $24 \mathrm{~h}$ in the presence or absence of escalating doses of DG013A. a After incubation, the uptake of latex beads coated with FITC-labeled rabbit IgG was measured. b Separately, cell media from RAW264.7 cells treated with LPS $(1 \mu \mathrm{g} / \mathrm{ml})$ and IFN $\gamma(100 \mathrm{IU} / \mathrm{ml})$ for $24 \mathrm{~h}$ were treated with escalating doses of DG013A as well as Leu-AMC. Enzymatic activity was determined by fluorescent measurement by

microplate reader. c IL-1 $\beta$ production by hPBMCs treated with ERAP1-KE in the presence or absence of $5 \mu \mathrm{M}$ of DG013A or DG002A was measured by ELISA. IL-6 (d) and TNFa (e) production levels by hPBMCs treated with ERAP1-KE in the presence or absence of $5 \mu \mathrm{M}$ of DG013A or DG002A were measured by ELISA. Data are representative of 2 independent experiments (c-e) from 2 different buffy coat donors. ${ }^{* * *} \mathrm{p}<0.001$ denote statistical significance from mock-treated cells.

crine effect of ERAP1 requires active enzymatic trimming abilities, we performed an L-AMC trimming assay, as previously described [13]. We observed that the secreted ERAP1 possesses potent active peptide trimming activities and that the use of DG013A significantly dampened these activities (fig. $4 \mathrm{~b}$ ).

To confirm the specific role of ERAP1 in mediating innate immunostimulatory responses, ERAP1-treated hPBMCs were mock-treated or pretreated with ERAP1 inhibitors (DG013A or DG002A) to quantify the production of IL-1 $\beta$, IL- 6 and TNF $\alpha$ by ELISA. Treatment of hPBMCs with ERAP1-RQ significantly induced IL-1 $\beta$ production (fig. 4c), which was partially abrogated in the presence of ERAP1 inhibitors. Supernatants derived from

these same cells were also utilized to evaluate IL- 6 and TNFa levels. Partial reductions in IL-6 levels were also observed following ERAP1 enzymatic activity inhibition (fig. 4d); however, TNFa production showed a complete dependence on the enzymatic activity of ERAP1 (fig. 4e). These data demonstrate that the enzymatic activity of ERAP1 is at least partially required for macrophage phagocytosis enhancement and inflammatory cytokine and chemokine induction by ERAP1-exposed hPBMCs.

\section{Mechanisms Underlying the Effect of ERAP1 on the}

Innate Immune Responses of Human PBMCs

We next wished to investigate the molecular mechanism by which ERAP1 induces innate immunity within 
human immune cells. In our previous experiments, we have noted that exposure of hPBMCs to the diseaselinked ERAP1 variants or treatment with ERAP1 highrisk-allele-expressing Ad5 vectors significantly increased IL- $1 \beta$ release. This led us to hypothesize that the diseaselinked ERAP1 variants might activate specific innate immune sensors which are responsible for mediating IL- $1 \beta$ processing and release, a mechanism that is strictly controlled by inflammasomes. NLRP3 (NOD-like receptor, pyrin domain-containing 3 ) is the most studied member of the inflammasome family [28]; we therefore investigated if NLRP 3 inflammasome controls some of the IL- $1 \beta$ production triggered by ERAP1. The NLRP3 inflammasome utilizes the proteolytic enzyme, caspase-1, to process IL-1 $\beta$ into its mature form [29]; we thereforeinvestigated if functional caspase- 1 is required for ERAP1 to induce IL-1 $\beta$ production. A caspase-1-specific peptide inhibitor, z-YVAD-FMK, was applied to hPBMCs, and we then evaluated IL- $1 \beta$ secretion in response to ERAP1 exposure. We found that the inhibition of caspase- 1 activity abolished the release of IL- $1 \beta$ (fig. $5 \mathrm{a}$ ), but not other cytokines (online suppl. fig. 3A, B), following ERAP1 treatment, compared to ERAP1-treated cells in the absence of z-YVAD-FMK (fig. 5a). As expected, inhibiting caspase- 1 activity had no effect on inflammatory cytokine production following LPS stimulation (online suppl. fig. 3C).

To investigate if ERAP1 was directly activating caspase-1, we measured activated caspase- 1 levels with a fluorescent cell-permeable probe that covalently binds only to activated caspase-1 [30]. Flow cytometry analysis revealed significant increases in caspase-1-positive cells after ERAP1-RQ stimulation when compared to mocktreated cells (online suppl. fig. 4).

To determine if the ERAP1/caspase-1 signaling pathway was responsible for the enhanced activation of specific human innate immune cells, we evaluated the impact of the caspase- 1 inhibitor on NK and DC cell function following ERAP1 exposure. Inhibition of caspase-1 activity completely abolished the ERAP1-RQ-mediated induction of CD107a expression on human NK cells when compared to cells treated with ERAP1-RQ in the absence of z-YVAD-FMK (fig. 5b). Similarly, CD80 expression on DCs was also induced by ERAP1-RQ treatment, while inhibiting caspase- 1 activity significantly reduced this response when compared to cells treated with ERAP1-RQ alone (fig. 5c). Overall, this suggests that ERAP1-RQ activates human NK cells and DCs in a mechanism that is, in part, dependent on an intact caspase-1 pathway.

Extracellular ERAP1 Activates Innate Immunity
Cathepsin-B-Dependent NLRP3 Inflammasome Induction, Cytokine Production and DC Activation by ERAP1 Variants

Cathepsin-B is involved in NLRP3 signaling, and the release of lysosomal cathepsin- $\mathrm{B}$ to the cytosol has been shown to induce the production of IL- $1 \beta$ as well as being involved in other aspects of NLRP3 inflammasome signaling [31]. To determine if ERAP1-induced IL-1 $\beta$ production may be influenced by the presence of cathepsin$B$, we examined if ERAP1 requires functional cathepsin- $B$ to induce IL- $1 \beta$ production. Human PBMCs were pretreated with the cathepsin-B-specific inhibitor, CA-074$\mathrm{Me}$ [29], and then exogenously exposed to ERAP1 variants. Consequentially, ERAP1-RQ and ERAP1-KE variants were found to induce potent IL- $1 \beta$ production in a cathepsin-B-dependent manner (fig. 5d). Similar results were also observed for ERAP1-RQ-mediated induction of IL- 6 and TNF $\alpha$ by hPBMCs (online suppl. fig. 5A, B). We also evaluated CD107 $\alpha$ expression on ERAP1-RQtreated human NK cells in the presence of CA-074-Me. The enhanced NK cell activation induced by ERAP1-RQ treatment was significantly $(\mathrm{p}<0.01)$ reduced following cathepsin-B inhibitor treatment (fig. 5e). We also evaluated CD80 expression on ERAP1-RQ-treated human DCs in the presence of CA-074-Me. Interestingly, CD80 expression was significantly induced by ERAP1-RQ, yet CA-074-Me significantly reduced this response (fig. 5f). Taken together, these data suggest the effects of diseasespecific ERAP1 variants on IL- $1 \beta$ release and activation of human immune cells requires functional cathepsin-B.

To investigate the potency of IL- $1 \beta$ induction by ERAP1, we compared it to two other potent NLRP3 activators: alum and ATP [28]. Human PBMCs were exposed to ERAP1-KE of high LPS concentration $(1 \mu \mathrm{g} / \mathrm{ml})$ or initially primed with a low LPS concentration $(25 \mathrm{pg} / \mathrm{ml})$, and exposed to the NLRP3 activators alum and ATP. Production of IL- $1 \beta$ was then evaluated by ELISA. Surprisingly, ERAP1-KE induced as high a concentration of IL$1 \beta$ as the known NLRP3 activators (fig. $5 \mathrm{~g}$ ), thus verifying the immunologic potency of ERAP1.

The efflux of cellular potassium is a common step shared by several NLRP3-activating stimuli and is required for NLRP3-dependent IL-1 $\beta$ release [32]. Moreover, past reports show that preventing potassium efflux by increasing the extracellular potassium concentration inhibits inflammasome activation and IL- $1 \beta$ release [33]. Consistent with these prior observations, we found that increasing extracellular potassium ablated the ERAP1RQ-mediated increase in IL- $1 \beta$ production by human cells when compared to cells treated with ERAP1-RQ 


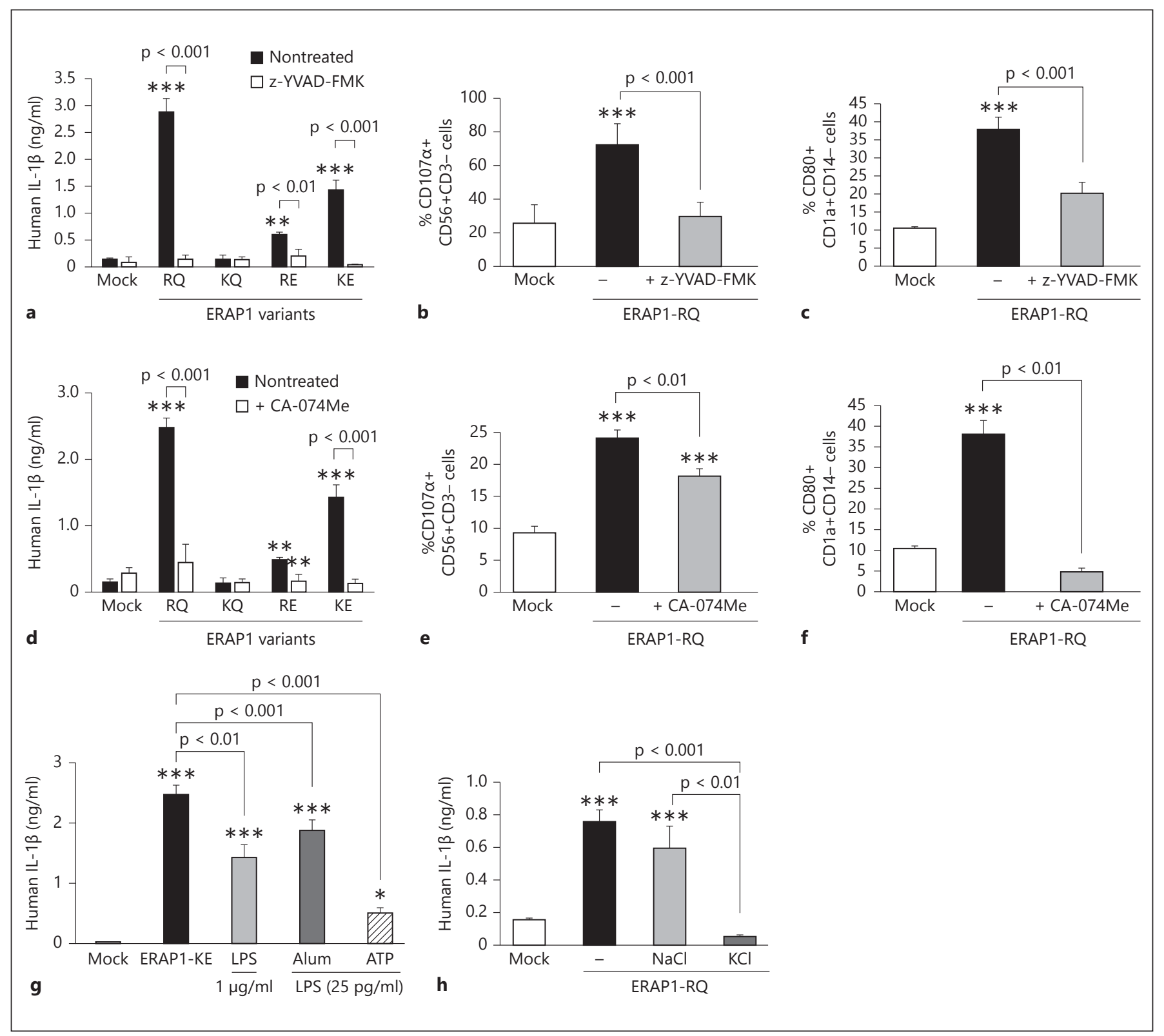

Fig. 5. ERAP1 requires intact caspase- 1 and cathepsin- $B$ to induce the innate immune responses of hPBMCs. a Human PBMC IL-1 $\beta$ production following ERAP1 treatment in the presence or absence of $10 \mu \mathrm{M}$ of $z$-YVAD-FMK. Expression of CD107a on NK cells (b) or CD80 on DCs (c) following ERAP1-RQ treatment in the presence or absence of $10 \mu \mathrm{M} z$-YVAD-FMK. d Human PBMC IL-1 $\beta$ production following ERAP1 treatment in the presence or absence of $10 \mu \mathrm{M}$ of CA-074Me. e Expression of CD107 $\alpha$ on ERAP1-RQtreated human NK cells in the presence of $10 \mu \mathrm{M}$ of CA-074Me. f Expression of CD80 on human DCs following ERAP1-RQ treatment in the presence or absence of CA-074Me. $g$ Human PBMCs were primed with LPS ( $25 \mathrm{pg} / \mathrm{ml})$ for $3 \mathrm{~h}$, then stimulated for an additional $48 \mathrm{~h}$ with alum $(500 \mu \mathrm{g} / \mathrm{ml})$, ATP $(5 \mathrm{mM})$ or a higher concentration of LPS $(1 \mu \mathrm{g} / \mathrm{ml})$, or else left unprimed and simply given ERAP1-KE. IL-1 $\beta$ secretion into the media was measured by ELISA. $\mathbf{h}$ Human PBMCs were either mock-treated or treated with ERAP1-RQ alone or ERAP1-RQ and media containing $15 \mathrm{mM}$ $\mathrm{NaCl}$ or $15 \mathrm{mM} \mathrm{KCl}$, and analyzed for IL-1 $\beta$ production at $48 \mathrm{~h}$ after treatment by ELISA. Data are representative of at least 5 (a-e) or $2(\mathbf{f}, \mathbf{g})$ independent experiments from different blood donors. ${ }^{*} \mathrm{p}<0.05,{ }^{* *} \mathrm{p}<0.01,{ }^{* * *} \mathrm{p}<0.001$ denote statistical significance compared to mock-treated cells. 


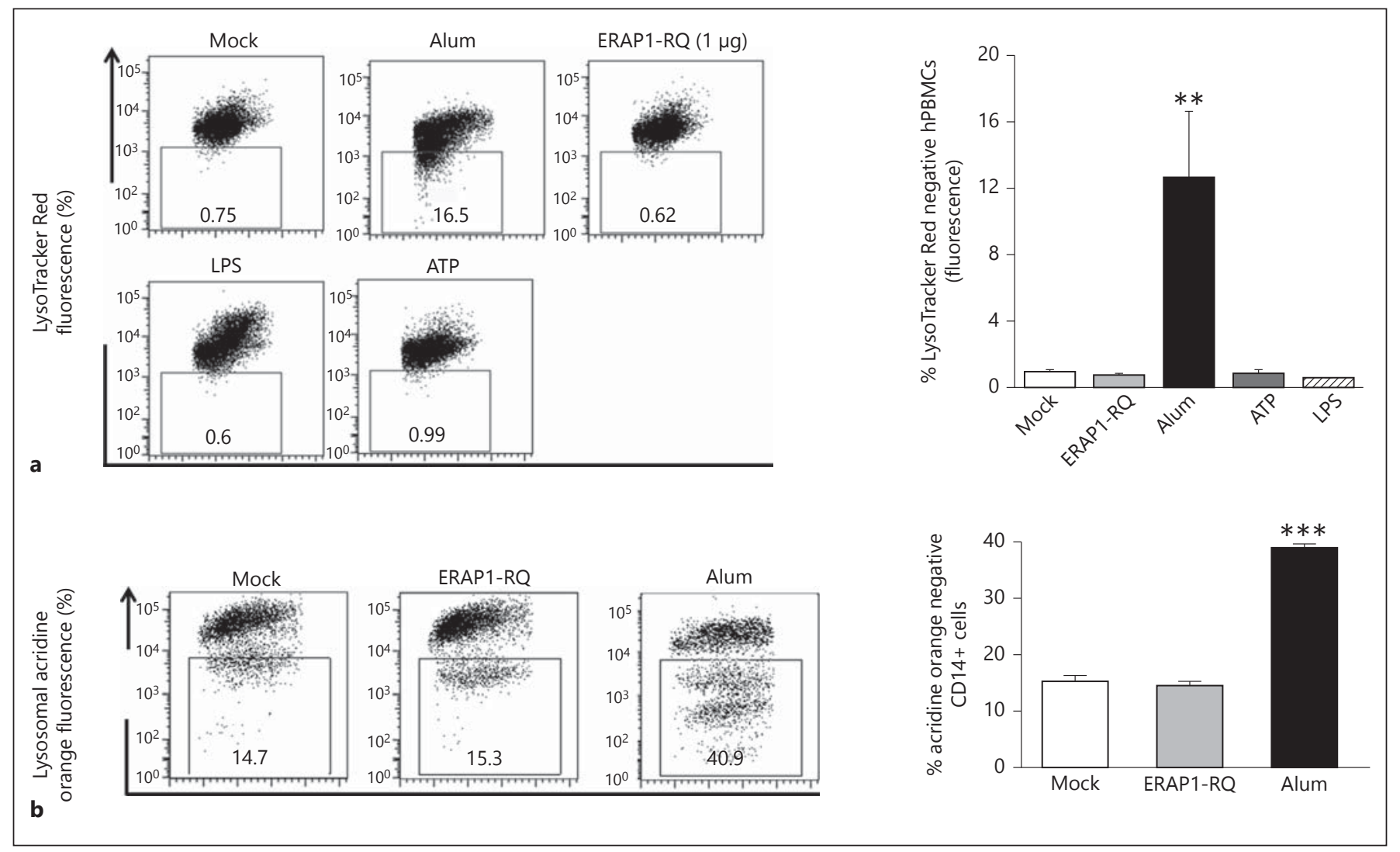

Fig. 6. ERAP1 protein does not induce IL-1 $\beta$ production via lysosomal destabilization. Human PBMCs were stained with the lysosomal staining dye, LysoTracker Red (150 nM; a) or acridine orange (b), and then treated for $18 \mathrm{~h}$ with $1 \mu \mathrm{g}$ ERAP1-RQ or alum $(500 \mu \mathrm{g} / \mathrm{ml})$, followed by flow cytometry analysis. The boxes within each figure contain a number indicating the percentage of cells lacking LysoTracker Red or acridine orange staining (excitation $488 \mathrm{~nm}$; emission 650-690 nm). Samples were plated in quadruplicate. Data are representative of 2 independent experiments from 2 different blood donors. ${ }^{* *} \mathrm{p}<0.01,{ }^{* * *} \mathrm{p}<0.001$ denote statistical significance compared to mock-treated cells. alone or ERAP1-RQ and a high $\mathrm{NaCl}$ concentration control (fig. 5h), indicating the specific involvement of potassium efflux in ERAP1-RQ-mediated NLRP3 activation. These results further suggest that ERAP1-RQ may exert activation effects via the NLRP3 pathway.

\section{ERAP1 Protein Induces IL-1 $\beta$ Production Independent} of Lysosomal Destabilization

Many NLRP3 inflammasome activators, such as alum, activate NLRP 3 and induce IL- $1 \beta$ release by destabilizing the lysosome and releasing cathepsin-B into the cytosol [34]. To explore the possibility that ERAP1 enhances IL- $1 \beta$ processing and release via a mechanism that involves lysosomal degradation, we evaluated lysosomal integrity following ERAP1-RQ treatment of hPBMCs. Cells were labeled with LysoTracker Red and lysosomal destabilization was evaluated, as previously described [35]. Human PBMCs were subsequently treated with ERAP1-RQ, alum, ATP or LPS, after which flow cytometry analysis was performed. Consistent with previously published reports, alum induced significant amounts of lysosomal rupture, evident from the decreased fluorescence of LysoTracker Red-labeled cells (fig. 6a). As expected, neither ATP, which induces NLRP3 inflammasome activation via potassium efflux [36], nor LPS treatment induced lysosomal destabilization. Interestingly, ERAP1-RQ did not induce lysosomal rupture, which was further confirmed by measuring lysosomal degradation with the lysosomal integrity monitoring dye, acridine orange ( $\mathrm{AO}$ ) (fig. 6b). These data suggest that, unlike alum, the disease-linked ERAP1-RQ variant induces IL- $1 \beta$ release in a mechanism that does not involve lysosomal rupture and may exert its effects prior to such events. 


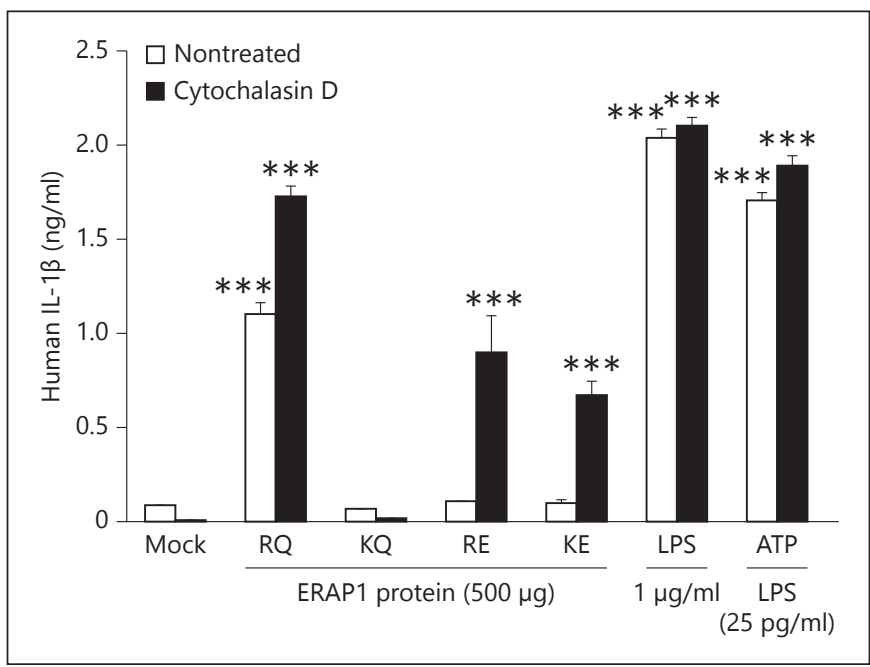

Fig. 7. Blocking phagocytosis of ERAP1 protein enhances IL-1 $\beta$ release: hPBMCs $\left(2 \times 10^{6}\right.$ cells/well $)$ were either mock-treated or treated with an ERAP1 variant in the presence or absence of $10 \mu \mathrm{M}$ cytochalasin D (added 30 min prior to ERAP1 stimulation). Culture media were collected at $48 \mathrm{~h}$ after treatment for ELISA measurement of IL- $1 \beta$ production. ${ }^{* * *} \mathrm{p}<0.001$ denotes statistical significance compared to mock-treated cells.

\section{Blocking Cellular Internalization of ERAP1 Protein}

Enhances IL-1 $\beta$ Release from $h P B M C s$

The phagocytosis of multiple NLRP3 inflammasome activators, such as bacterial toxins and several crystals, is an essential mechanism of NLRP3 inflammasome activation [31]. We investigated whether cellular internalization of ERAP1 protein is required for IL- $1 \beta$ release by hPBMCs, by treating them with the actin polymerization inhibitor cytochalasin $\mathrm{D}$, a potent inhibitor of phagocytosis, and then exogenously exposing the cells to ERAP1 variants. ELISA measurement of the production of IL- $1 \beta$ revealed that inhibiting ERAP1 endocytosis effectively increased IL- $1 \beta$ secretion (fig. 7 ), suggesting that ERAP1 has potent extracellular innate immune activities and that cellular uptake of ERAP1 not necessary for it to activate human immune cells. We also found that the diseaselinked ERAP1 variant, ERAP1-RQ, induces more potent IL-1 $\beta$ release than other ERAP1 variants. Furthermore, exposing hPBMCs to the protective ERAP1 variant, ERAP1-KQ, did not induce IL- $1 \beta$ production. IL- $1 \beta$ production triggered by LPS or extracellular ATP, which utilize the TLR4 and P2X7 receptor, respectively (and not the phagocytic machinery), to activate the NLRP3 inflammasome, remained largely unaffected by cytochalasin D treatment, confirming that the cells maintained an intact NLRP3 inflammasome pathway in the presence of an en- docytosis inhibitor at these doses (fig. 7). These data suggest that disease-linked ERAP1 variants have both extracellular and intracellular pathways contributing to their stimulatory innate immune responses within human cells.

\section{Discussion}

Multiple ERAP1 polymorphisms have been implicated in the pathogenesis of several autoimmune diseases [7, $9,10]$. ERAP1 plays a canonical role in MHC class I antigen presentation. It has been widely hypothesized that its role in the pathogenesis of autoimmune disorders was primarily mediated by this, in addition to its potential downstream effects on CD8+ T cell responses [7]. Our previous studies and those of other groups indirectly suggested a potential role for ERAP1 in modulating innate immune responses and that these may also play a role in disease pathogenesis $[7,16,26]$. However, the exact molecular mechanism behind these phenomena has not been elucidated.

In this report, we have found that extracellular ERAP1 potently activates both innate and adaptive immune components in hPBMC populations. In addition, our results suggest that these responses are, in part, mediated by the caspase-1/NLRP3 inflammasome pathway. Our data suggest that ERAP1-mediated NLRP3 inflammasome activation is required for inducing IL- $1 \beta$ production and activating NK, DC and T cells. Our data also reveal that the cellular internalization of ERAP1 protein is not required for IL- $1 \beta$ release, suggesting that extracellular ERAP1 protein can activate this innate immune pathway in hPBMCs, independent of internalization. Our data demonstrate that ERAP1 induces IL-1 $\beta$ in a mechanism that is completely dependent on a functional caspase-1 pathway. However, it is important to note that inhibiting caspase- 1 activity did not impact the release of several other cytokines and chemokines that we found were induced by the ERAP1 protein; this promotes our hypothesis that ERAP1 may interact with and activate multiple distinct pathways to mediate these immune responses. Since caspase- 1 processing and activation can be induced by several other caspase-1-activating inflammasomes (e.g. AIM2, NLRP1b, NLRC4 and NLRP6) [28], it is possible that they may also have a role in ERAP1 innate immunostimulatory activities. In addition, since the caspase- 1 inhibitor that we utilized can also interfere with caspase- 4 and caspase- 5 activities, it is possible that they may also have a role in 
ERAP1 innate immunostimulatory activities. Future studies are now required to discern these intriguing complexities.

Recently, it has been shown that the cytoplasm-localized inflammasome adaptor ASC can be secreted extracellularly, allowing it to be internalized and function as a danger signal that induces NLRP3/caspase- 1 activation and IL- $1 \beta$ processing and release $[37,38]$. These data are in parallel to our findings by suggesting that the release of intracellular proteins like ERAP1 into the extracellular environment can trigger the NLRP3 inflammasome and possibly other innate immune pathways.

We also found that exogenous provision of specific, disease-linked ERAP1 variants (ERAP-RQ and ERAP1$\mathrm{KE}$ ) induced more potent inflammatory cytokine and chemokine responses by hPBMCs than other ERAP1 variants (e.g. ERAP1-KQ), specifically, IL-1 $\beta$, IL-6 and TNFa. The exact molecular mechanisms underlying the observed differences in innate immune stimulation activities between ERAP1 variants is not clear at this time; however, the presence of specific ERAP1 SNPs may alter critical aspects of ERAP1 protein structure and may result in several nonmutually exclusive outcomes, including the induction of a misfolded protein response, a mechanism that has been shown previously to activate the NLRP3 inflammasome pathway [39]. In addition, the ERAP1 K528R SNP is located at a critical domain junction, and has been proposed to affect both the specificity and the enzymatic activity of the protein, possibly by affecting interdomain interactions [40]. In contrast, the Q730E SNP is located at the inner surface of the C-terminal cavity of ERAP1, a location that might affect the substrate sequence or length specificities of the aminopeptidase. Since ERAP1 enzymatic activity is required for innate immune stimulation of hPBMCs, ERAP1 SNPs that impact upon its enzymatic activity may also have a direct impact on the innate immune stimulation function of ERAP1.

These novel results may shed light upon past genomewide association studies findings that demonstrated an association between specific ERAP1 variants and the development of certain autoimmune diseases [7], as several studies have also shown that these patients have increased serum levels of these inflammatory cytokines [41]. More specifically, AS patients have been shown to have increased plasma concentrations of IL- $1 \beta$ and TNF $\alpha$, while the use of IL-1R and TNFa antagonists [42] have been shown to reduce AS symptoms.

In previous studies [14], we used ERAP1 variants containing multiple AS-associated SNPs [18] and identified that specific variants of ERAP1 protein function to differentially regulate peptide trimming and surface expression of antigenic peptides presented by HLA-B27 molecule [18]. Here, we confirm our previous findings and provide additional evidence that different ERAP1 alleles differentially impact on ERAP1-mediated functions. Specifically, we found that overexpression of two structurally different ERAP1 variants induced different innate immune profiles mediated by hPBMCs. However, our current data suggest that utilizing ERAP1 proteins containing two SNPs (ERAP1-KQ and ERAP1-RE) does not result in innate immune stimulation within human immune cells that is as potent as treatment with ERAP1 variants harboring a single SNP. For example, the introduction of a single disease-associated SNP into the ERAP1 protein sequence, e.g. ERAP1-K528R (ERAP1-RQ) and ERAP1Q730E (ERAP1-KE), significantly increased the ability of these ERAP1 protein variants to stimulate innate immune responses by human cells.

In addition, we found that the innate immune responses of hPBMCs induced by treatment with the ERAP1-KQ variant are minimal. Mutating a single amino acid, K528R (ERAP1-RQ) or Q730E (ERAP1-KE), resulted in a complete shift of the innate immune function of these ERAP1 protein variants. In contrast, combining the two immunostimulatory residues of ERAP1, K528R and Q730E (ERAP1-RE) resulted in a moderate induction of the innate immune responses of hPBMCs when exposed to this particular ERAP1 variant. These data suggest the presence of a negative synergy between the ERAP1-KQ and ERAP1-RE mutations.

As ERAP1 expression has been shown to be increased in certain cancers, the role of ERAP1 in the innate immune system $[16,26]$ may also be a factor in tumor development and progression [43]. Several types of tumors often overexpress proinflammatory mediators that can enhance tumor progression [44], including TNFa and IL-6 [44], both of which we demonstrated are induced by Ad-based ERAP1 overexpression. Our data also reveal the potent activation of several human innate immune cell types by disease-linked ERAP1 variants. Specifically, we found that ERAP1-RQ induced the potent degranulation of human NK cells, an innate immune cell subset that has been shown to play major roles in the development of several autoimmune diseases.

A wide range of pathogenic, endogenous or environmental stimuli including ATP, bacterial toxins and salt crystals activate the NLRP3 inflammasome [28], which in turn stimulates the production of IL-1 $\beta$, a key mediator of acute and chronic inflammation [1]. Here, we have also 
shown that disease-associated ERAP1 variants are capable of enhancing IL- $1 \beta$ production when exogenously provided to hPBMCs via the activation of the caspase- 1 and NLRP3 pathways. Previous studies show that decreased cytosolic potassium correlates with NLRP3-dependent, caspase- 1 activation [32,33], and that preventing potassium efflux by increasing its extracellular concentration inhibits adjuvant stimulation of NLRP3 activation [35]. In this report, we have shown that increasing extracellular potassium completely abrogated IL-1 $\beta$ production from ERAP1-RQ-treated hPBMCs, suggesting that activation of the NLRP3 inflammasome occurs after ERAP1-RQ exposure. ERAP1 enzyme-chelating agents such as EDTA or low-weight ERAP1 inhibitors both abolished ERAP1-mediated IL- $1 \beta$ production, further suggesting that ERAP1 enzymatic activity is critical for its activation of NLRP3 and stimulation of IL- $1 \beta$ production.

The release of lysosomal cathepsin-B has been implicated in NLRP3 activation [31], and we have found that functional cathepsin-B is critical for the ERAP1-mediated enhancement of innate immune responses of hPBMCs. Furthermore, we found that inhibiting caspase- 1 activity completely abolished IL- $1 \beta$ production after exogenous ERAP1 exposure of hPBMCs, but not other ERAP1-induced proinflammatory cytokines, signifying that ERAP1 might activate multiple signaling pathways.
The identification of ERAP1 as a potent activator of the NLRP3 inflammasome suggests that it may be contributing to the pathogenesis of autoimmune diseases via this innate immune pathway, in addition to its canonical role in antigen presentation. Future studies that include characterizing the molecular mechanism of the impact of ERAP1 on human innate immune cells as well as adaptive immune cells may clarify the association of ERAP1 and autoimmune diseases.

\section{Acknowledgement}

We wish to thank the Michigan State University Laboratory Animal Support Facility for their assistance in the humane care and maintenance of the animals utilized in this work. A.A. was supported by a National Institutes of Health grant 5R01 AR056981, the Michigan State University Foundation and the Osteopathic Heritage Foundation. E.S. acknowledges the support from the European Union (European Social Fund) and Greek national funds through the Operational Program 'Education and Lifelong Learning' of the National Strategic Reference Framework - Research Funding Program of the General Secretariat for Research and Technology (grant ERC-14).

\section{Disclosure Statement}

The authors declare no competing financial interests.

\section{References}

1 Kastner DL, Aksentijevich I, Goldbach-Mansky R: Autoinflammatory disease reloaded: a clinical perspective. Cell 2010;140:784-790.

-2 Doria A, Zen M, Bettio S, Gatto M, Bassi N, Nalotto L, Ghirardello A, Iaccarino L, Punzi L: Autoinflammation and autoimmunity: bridging the divide. Autoimmun Rev 2012;12: 22-30.

3 Danzer C, Mattner J: Impact of microbes on autoimmune diseases. Arch Immunol Ther Exp (Warsz) 2013;61:175-186.

4 O'Neill LA, Bowie AG: Sensing and signaling in antiviral innate immunity. Curr Biol 2010; 20:R328-R333.

5 Medzhitov R, Horng T: Transcriptional control of the inflammatory response. Nat Rev Immunol 2009;9:692-703.

6 Netea MG, Wijmenga C, O’Neill LA: Genetic variation in Toll-like receptors and disease susceptibility. Nat Immunol 2012;13:535-542.

7 Fierabracci A, Milillo A, Locatelli F, Fruci D: The putative role of endoplasmic reticulum aminopeptidases in autoimmunity: insights from genomic-wide association studies. Autoimmun Rev 2012;12:281-288.
8 Tsujimoto M, Hattori A: The oxytocinase subfamily of M1 aminopeptidases. Biochim Biophys Acta 2005;1751:9-18.

-9 Yamamoto N, Nakayama J, Yamakawa-Kobayashi K, Hamaguchi H, Miyazaki R, Arinami T: Identification of 33 polymorphisms in the adipocyte-derived leucine aminopeptidase (ALAP) gene and possible association with hypertension. Hum Mutat 2002;19:251-257.

10 Keidel S, Chen L, Pointon J, Wordsworth P: ERAP1 and ankylosing spondylitis. Curr Opin Immunol 2013;25:97-102.

11 Reeves E, Edwards CJ, Elliott T, James E: Naturally occurring ERAP1 haplotypes encode functionally distinct alleles with fine substrate specificity. J Immunol 2013;191:35-43.

12 Nguyen TT, Chang SC, Evnouchidou I, York IA, Zikos C, Rock KL, Goldberg AL, Stratikos E, Stern LJ: Structural basis for antigenic peptide precursor processing by the endoplasmic reticulum aminopeptidase ERAP1. Nat Struct Mol Biol 2011;18:604-613.

13 Evnouchidou I, Kamal RP, Seregin SS, Goto Y, Tsujimoto M, Hattori A, Voulgari PV, Drosos AA, Amalfitano A, York IA, Stratikos
E: Cutting edge: coding single nucleotide polymorphisms of endoplasmic reticulum aminopeptidase 1 can affect antigenic peptide generation in vitro by influencing basic enzymatic properties of the enzyme. J Immunol 2011;186:1909-1913.

14 Seregin SS, Rastall DP, Evnouchidou I, Aylsworth CF, Quiroga D, Kamal RP, GodbehereRoosa S, Blum CF, York IA, Stratikos E, Amalfitano A: Endoplasmic reticulum aminopeptidase-1 alleles associated with increased risk of ankylosing spondylitis reduce HLA-B27 mediated presentation of multiple antigens. Autoimmunity 2013;46:497-508.

15 Hammer GE, Gonzalez F, Champsaur M, Cado $\mathrm{D}$, Shastri N: The aminopeptidase ERAAP shapes the peptide repertoire displayed by major histocompatibility complex class I molecules. Nat Immunol 2006;7:103-112.

-16 Aldhamen YA, Seregin SS, Rastall DP, Aylsworth CF, Pepelyayeva Y, Busuito CJ, Godbehere-Roosa S, Kim S, Amalfitano A: Endoplasmic reticulum aminopeptidase-1 functions regulate key aspects of the innate immune response. PLoS One 2013;8:e69539. 
$\checkmark 17$ Aldhamen YA, Appledorn DM, Seregin SS, Liu CJ, Schuldt NJ, Godbehere S, Amalfitano A: Expression of the slam family of receptors adapter EAT-2 as a novel strategy for enhancing beneficial immune responses to vaccine antigens. J Immunol 2011;186:722-732.

- 18 Seregin SS, Rastall DP, Evnouchidou I, Aylsworth CF, Quiroga D, Kamal RP, Godbehere-Roosa S, Blum CF, York IA, Stratikos E, Amalfitano A: Endoplasmic reticulum aminopeptidase-1 alleles associated with increased risk of ankylosing spondylitis reduce HLA-B27 mediated presentation of multiple antigens. Autoimmunity 2013;46:497-508.

-19 Hartman ZC, Osada T, Glass O, Yang XY, Lei GJ, Lyerly HK, Clay TM: Ligand-independent toll-like receptor signals generated by ectopic overexpression of MYD88 generate local and systemic antitumor immunity. Cancer Res 2010;70:7209-7220.

-20 Osada T, Yang XY, Hartman ZC, Glass O, Hodges BL, Niedzwiecki D, Morse MA, Lyerly HK, Amalfitano A, Clay TM: Optimization of vaccine responses with an E1, E2b and E3-deleted Ad5 vector circumvents pre-existing anti-vector immunity. Cancer Gene Ther 2009; 16:673-682.

- 21 Aldhamen YA, Seregin SS, Aylsworth CF, Godbehere S, Amalfitano A: Manipulation of EAT-2 expression promotes induction of multiple beneficial regulatory and effector functions of the human innate immune system as a novel immunomodulatory strategy. Int Immunol 2014;26:291-303.

-22 Aylsworth CF, Aldhamen YA, Seregin SS, Godbehere S, Amalfitano A: Activation of human natural killer cells by the novel innate immune modulator recombinant Eimeria antigen. Hum Immunol 2013;74:916-926.

$\checkmark 23$ Goto Y, Hattori A, Ishii Y, Tsujimoto M: Reduced activity of the hypertension-associated Lys528Arg mutant of human adipocyte-derived leucine aminopeptidase (A-LAP)/ERaminopeptidase-1. FEBS Lett 2006;580:18331838.

-24 Zervoudi E, Papakyriakou A, Georgiadou D, Evnouchidou I, Gajda A, Poreba M, Salvesen GS, Drag M, Hattori A, Swevers L, Vourloumis D, Stratikos E: Probing the s1 specificity pocket of the aminopeptidases that generate antigenic peptides. Biochem J 2011;435:411-420.

-25 Zervoudi E, Saridakis E, Birtley JR, Seregin SS, Reeves E, Kokkala P, Aldhamen YA, Amalfitano A, Mavridis IM, James E, Georgiadis D, Stratikos E: Rationally designed inhibitor targeting antigen-trimming aminopeptidases enhances antigen presentation and cytotoxic T-cell responses. Proc Natl Acad Sci U S A 2013;110:19890-19895.
6 Goto Y, Ogawa K, Hattori A, Tsujimoto M: Secretion of endoplasmic reticulum aminopeptidase 1 is involved in the activation of macrophages induced by lipopolysaccharide and interferon-gamma. J Biol Chem 2011; 286:21906-21914.

27 Schomburg L, Kollmus H, Friedrichsen S, Bauer K: Molecular characterization of a puromycin-insensitive leucyl-specific aminopeptidase, PILS-AP. Eur J Biochem 2000;267: 3198-3207.

28 Rathinam VA, Vanaja SK, Fitzgerald KA: Regulation of inflammasome signaling. Nat Immunol 2012;13:333-342.

29 Martinon F, Burns K, Tschopp J: The inflammasome: a molecular platform triggering activation of inflammatory caspases and processing of proIL-beta. Mol Cell 2002;10:417426.

- 30 Amstad PA, Yu G, Johnson GL, Lee BW, Dhawan S, Phelps DJ: Detection of caspase activation in situ by fluorochrome-labeled caspase inhibitors. Biotechniques 2001;31:608-614.

31 Hornung V, Bauernfeind F, Halle A, Samstad EO, Kono H, Rock KL, Fitzgerald KA, Latz E: Silica crystals and aluminum salts activate the NALP3 inflammasome through phagosomal destabilization. Nat Immunol 2008;9:847856.

32 Munoz-Planillo R, Kuffa P, Martinez-Colon G, Smith BL, Rajendiran TM, Nunez G: K(+) efflux is the common trigger of NLRP3 inflammasome activation by bacterial toxins and particulate matter. Immunity 2013;38: 1142-1153.

33 Petrilli V, Papin S, Dostert C, Mayor A, Martinon F, Tschopp J: Activation of the NALP3 inflammasome is triggered by low intracellular potassium concentration. Cell Death Differ 2007;14:1583-1589.

34 Kuroda E, Ishii KJ, Uematsu S, Ohata K, Coban C, Akira S, Aritake K, Urade Y, Morimoto Y: Silica crystals and aluminum salts regulate the production of prostaglandin in macrophages via NALP3 inflammasome-independent mechanisms. Immunity 2011;34:514526.

35 Eisenbarth SC, Colegio OR, O'Connor W, Sutterwala FS, Flavell RA: Crucial role for the NALP3 inflammasome in the immunostimulatory properties of aluminium adjuvants. Nature 2008;453:1122-1126.
6 Perregaux D, Gabel CA: Interleukin-1 beta maturation and release in response to ATP and nigericin. Evidence that potassium depletion mediated by these agents is a necessary and common feature of their activity. J Biol Chem 1994;269:15195-15203.

37 Baroja-Mazo A, Martin-Sanchez F, Gomez AI, Martinez CM, Amores-Iniesta J, Compan V, Barbera-Cremades M, Yague J, RuizOrtiz E, Anton J, Bujan S, Couillin I, Brough $D$, Arostegui JI, Pelegrin P: The NLRP3 inflammasome is released as a particulate danger signal that amplifies the inflammatory response. Nat Immunol 2014;15:738748.

38 Franklin BS, Bossaller L, De Nardo D, Ratter JM, Stutz A, Engels G, Brenker C, Nordhoff M, Mirandola SR, Al-Amoudi A, Mangan MS, Zimmer S, Monks BG, Fricke M, Schmidt RE, Espevik T, Jones B, Jarnicki AG, Hansbro PM, Busto P, Marshak-Rothstein A, Hornemann S, Aguzzi A, Kastenmuller W, Latz E: The adaptor ASC has extracellular and 'prionoid' activities that propagate inflammation. Nat Immunol 2014;15:727-737.

39 Masters SL, O’Neill LA: Disease-associated amyloid and misfolded protein aggregates activate the inflammasome. Trends Mol Med 2011;17:276-282.

40 Kochan G, Krojer T, Harvey D, Fischer R, Chen L, Vollmar M, von Delft F, Kavanagh KL, Brown MA, Bowness P, Wordsworth P, Kessler BM, Oppermann U: Crystal structures of the endoplasmic reticulum aminopeptidase-1 (ERAP1) reveal the molecular basis for N-terminal peptide trimming. Proc Natl Acad Sci U S A 2011;108:7745-7750.

41 Fung EY, Smyth DJ, Howson JM, Cooper JD, Walker NM, Stevens H, Wicker LS, Todd JA: Analysis of 17 autoimmune disease-associated variants in type 1 diabetes identifies 6q23/ TNFAIP3 as a susceptibility locus. Genes Immun 2009;10:188-191.

42 Bennett AN, Tan AL, Coates LC, Emery P, Marzo-Ortega H, McGonagle D: Sustained response to anakinra in ankylosing spondylitis. Rheumatology (Oxford) 2008;47:223224.

43 Fruci D, Ferracuti S, Limongi MZ, Cunsolo V, Giorda E, Fraioli R, Sibilio L, Carroll O, Hattori A, van Endert PM, Giacomini P: Expression of endoplasmic reticulum aminopeptidases in EBV-B cell lines from healthy donors and in leukemia/lymphoma, carcinoma, and melanoma cell lines. J Immunol 2006;176: 4869-4879.

44 Candido J, Hagemann T: Cancer-related inflammation. J Clin Immunol 2013;33(suppl 1): S79-S84.
Extracellular ERAP1 Activates Innate Immunity
J Innate Immun 2015;7:275-289 DOI: $10.1159 / 000368899$ 